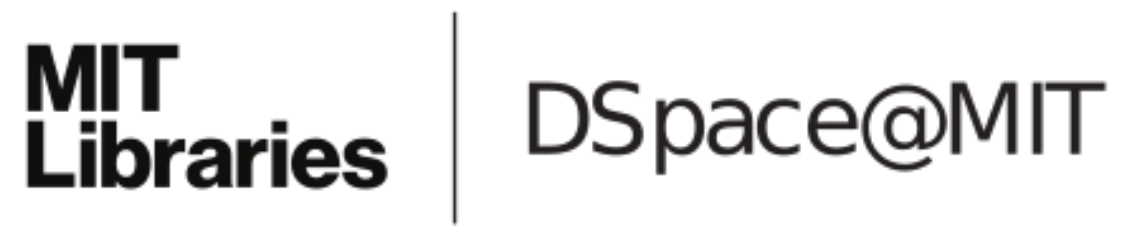

\author{
MIT Open Access Articles
}

\section{A Taxonomy for Characterizing Modes of Interactions in Goal-driven, Human-robot Teams}

The MIT Faculty has made this article openly available. Please share how this access benefits you. Your story matters.

Citation: Parashar, Priyam, Sanneman, Lindsay M., Shah, Julie A and Christensen, Henrik I. 2020. "A Taxonomy for Characterizing Modes of Interactions in Goal-driven, Human-robot Teams." IEEE International Conference on Intelligent Robots and Systems.

As Published: http://dx.doi.org/10.1109/IR0S40897.2019.8967974

Publisher: Institute of Electrical and Electronics Engineers (IEEE)

Persistent URL: https://hdl.handle.net/1721.1/137316

Version: Author's final manuscript: final author's manuscript post peer review, without publisher's formatting or copy editing

Terms of use: Creative Commons Attribution-Noncommercial-Share Alike 


\title{
A Taxonomy for Characterizing Modes of Interactions in Goal-driven, Human-robot Teams
}

\author{
Priyam Parashar ${ }^{1}$, Lindsay M. Sanneman ${ }^{2}$, Julie A. Shah ${ }^{2}$, Henrik I. Christensen ${ }^{1}$
}

\begin{abstract}
As robots and other autonomous agents are increasingly incorporated into complex domains, characterizing interaction within heterogeneous teams that include both humans and machines becomes more necessary. Previous literature has addressed the task of characterizing human-robot interaction from different perspectives and in multiple contexts. However, the numerous factors behind interaction work in conjunction, and the insights gained from one perspective can inadvertently affect another, creating a need for unification of these taxonomies and frameworks within an overarching taxonomy that systematically defines these relationships. In this paper we review existing taxonomies related to human-robot interaction, the behavioral sciences, and social and algorithmic taxonomies, and propose an overarching ontology for the factors from these works. We identify three main components characterizing the structure of an interaction (environment, task, and team), and structure them over two levels: contextual factors and factors driven by local dynamics. Finally, we present an analysis of how these factors affect decisions about levels of robot automation and level of information abstraction in an interaction, and discuss curent gaps in the literature that can motivate future research.
\end{abstract}

\section{INTRODUCTION}

The applicability of robotics has extended beyond a single autonomous agent interacting with a single human to scenarios involving larger heterogeneous teams of multiple, functionally different agents and humans working together ([1]). Furthermore, these interactions are occurring in more dynamic and less regulated environments which fundamentally impacts considerations at the design and development level with regard to human-robot team stability. Existing taxonomies characterize individual factors that impact interaction within in these increasingly complex domains ([2][8]), but none comprehensively cover the characterization of the operational space. Furthermore, each existing taxonomy maintains its own set of assumptions, which in turn limits the scope of a given taxonomy's applicability. In this paper, we combine individual taxonomies into a single overarching taxonomy with the aim of standardizing the nature of the interactions in question. This helps to methodically characterize interactions and to highlight both the benefits and drawbacks of an overall system. The gaps in existing literature to characterize the various nuances of operational

\footnotetext{
1 Contextual Robotics Institute, University of California, San Diego pparasha, hichristensen@eng.ucsd.edu

2 Interactive Robotics Group, MIT, Cambridge lindsays, julie_a_shahecsail.mit.edu

This work was supported by the DCIST Collaborative Research Alliance at the Army Research Lab
}

contexts in HRI are demonstrated by urban search and rescue (USAR) and assembly line use case scenarios in Section III

The primary aim of this paper is to propose a unified overarching taxonomy with the dual purpose of preserving the characterization of interactions within the surveyed taxonomies while also allowing for extension of their structures for increasingly complex scenarios. Through a review of existent taxonomies, we identified specific factors that impact team interactions, and have grouped these factors into three overarching themes that form the main structure of our overarching taxonomy. Additionally, we present an analysis of operational contexts relevant to HRI in order to highlight the degree of complexities introduced by the interplay and variability of these factors. The body of literature we drew from included works exploring interaction within the behavioral sciences $([9]-[11])$ and in social contexts $([5],[12])$, as well as the algorithmic techniques driving interaction in humanrobot teams $([2],[7])$. In this paper, we propose a framework that systematically assimilates surveyed taxonomies to more comprehensively classify factors that impact interaction in cooperative mixed teams working toward a joint goal, and highlight areas where this framework could be extended in the future. However, such a discussion can envelope numerous taxonomies including those which characterize affective factors of an exchange or characterize various interfaces between the agents. In order to scope the analysis, we limit current discussion to the structure and content rather than the medium and pattern of communication.

\section{AN OVERVIEW OF EXISTENT TAXONOMIES IN HRI}

The taxonomies we surveyed addressed distinct aspects of a cooperative system, including various factors related to task context and team configuration. Further, these taxonomies differed with regard to the level of abstraction of the factors under consideration, both over the breadth of factors, such as task context ([2]) and team configuration ([3], [4]]), and also over depth, from macro-context $([\overline{8}])$ to local complexities (or dynamics) of a specific task instantiation ([7], [13]). Additionally, each taxonomy describes interactions within a specific environmental context. Thus we identified a gap in terms of characterization of the environmental context, which is instrumental to characterizing the challenges to the system in the operational use cases detailed in Section III We also identified overlapping taxonomies that analyze effects of similar factors but from different perspectives. These can be combined for a more comprehensive view of interaction. 


\section{A. Context-driven Taxonomies}

We define contextual taxonomies as those describing the "problem" and "solution" contexts of an HRI application. In this case, the kind of task being undertaken and the operational requirements for accomplishing the task form the "problem" component, while the team configuration and capabilities are identified as the "solution."

1) Task and Environment as the Problem: Yanco and Drury ([8]) provided a meta-survey identifying factors that are critical to our definition of interaction "context (Section V). However, the paper itself does not impose any structure over these dimensions, even though they differ significantly in the level of system characterization. For example, while task type is a context-sensitive variable, centralized versus decentralized modes of command consensus represents a local factor impacted by the particular role that interactants play within a teams hierarchy. Furthermore, many of the variables specific to local dynamics are affected by contextlevel variables, so in addition to identifying important factors for interaction, it is equally important to elucidate the relationships between those factors. Recently, Beer, Rieth, Tran, et al. addressed the problem of classifying mixedteam HRI task contexts and their effects on inter-team interactions for military and commercial applications ([2]). While the authors maintained an explicit division between task and team model, the environment was relegated to the second level of categorization and only considered in the context of the constraints (speed, stealth, et cetera) it placed on the system. Beer, Rieth, Tran, et al. ([2]) additionally identified six main task focuses for missions undertaken within military and commercial HRI contexts, which we extend and incorporate into our own overarching taxonomy in Section $\mathrm{V}$

In the context of social robotics, Dautenhahn considered how to evaluate the extent of social skill required for HRI applications in different domains ([14]). The authors used four major axes to evaluate HRI application areas: contact with humans, degree of robot functionality, the role of the robot, and the necessity of social skills for success given the operational context. Our taxonomy incorporates these insights by merging the contact-frequency and social-skillrequirement axes. The role axis mentioned by Dautenhahn is subsumed by the general human-robot-roles theory in HRI ([15]), which is also included within our framework. Further, Phillips, Schaefer, Billings, et al. took inspiration from human-animal cooperation and characterized cooperative activities as physical, emotional (or, more generally, social), and cognitive ([16]).

2) Collective or Solution Specification: We define a collective as a heterogeneous team of humans and robots. In a collective, the team hierarchy can be flat or not, and the robotic agents can also be heterogeneous. Heterogeneity in agents is characterized by differences in terms of capabilities, possible actions and knowledge-base ([17]), among other factors. The team context specifies macro-dimensions, including the size and composition of the group ([2]) and communication constraints imposed by system design ([3], [4]). Another important aspect of characterizing a team is the a priori modeling of teammates in order to better understand their needs during task execution. Different methodologies exist like defining the belief-desire-intent of agents ([3]|) or their goals, actions, and knowledge structures ([17]).

\section{B. Cooperation-driven Taxonomies}

The humans role is one of the most important behavioral factors shaping human-robot interaction. Scholtz ([15]) used Norman's seven-stage model of interaction design ([18]) to reflect upon the possible levels of failure in a robot's design and to typify the subsequently necessary assistance according to the intermediate layers of intention, action, and perception of the agent. This led to the formulation of the five archetypal roles humans can play in order to assist the robot during HRI: 1) supervisor, 2) operator, 3) mechanic, 4) peer, and 5) bystander. Norman used this model to conceptualize human-centric design such that design of an artifact supports human understanding, but Scholtz uses the model to instead consider a robot-focused view of the usefulness of human knowledge such that the combined human-robot system is usable. Scholtz's set of roles was most recently updated with two additional entries: "information consumer and "mentor, the latter of which is specific to robots ([5]).

The literature explains why such roles are necessary for a usable system and presents a detailed synopsis of what information system designers should focus on while creating a human-robot interface to fill these roles. However, as we move toward a more collaborative nature of HRI, autonomous agents will increasingly be required to fluidly move through a team hierarchy and perform different roles in different contexts, just as we expect from a collaborative team of humans. Therefore, it will be important to define the expertise and knowledge required for each role and ground them within the mission context and corresponding team knowledge structure, enabling autonomous agents to effectively transition between roles.

In contexts where social interactions are interwoven with team functionality, Fong, Nourbakhsh, and Dautenhahn, FeilSeifer and Matarić, and Dautenhahn presented taxonomies identifying the basic components of a robotic system that affect human-robot social interactions ([12], [14], [19]). We incorporate these considerations into our own, more generalized framework. We also note the inclusion of a training phase for interactions ([12]) in prior task taxonomies, and merge this additional aspect with the task focus list ([2]) in our own taxonomy. Explicit user-type modeling has benefited HRI systems but most of these observations are from contexts where the human and robot remain fixed in a role for the entirety of the interaction; there is still a need for deeper discussion about the types and transitions between roles humans and robots can afford in contexts where team hierarchy is more fluid ([14], [19]).

1) Information requirements for cooperation: In order to study information exchange during interactions, it is necessary to first analyze the question of what role the 
given information has, and why it must be disseminated in the given context. Endsley introduced a framework that defined the concept of "situational awareness" according to the processing mechanisms, design, and knowledge of a dynamic system ([10]). This framework delineates information into three progressively complex levels comprising an individual's situational awareness (SA). Chen, Lakhmani, Stowers, et al. formally define the components defining a mission's description as well as the required knowledgestructures, and qualitatively ground the SA model using these concepts, calling it a Situation Awareness-Based Agent Transparency (SAT) model ([9]). The following are the levels of SA defined by Endsley that are incorporated into the SAT model: • Level 1 - Perception. - Level 2 - Comprehension 1 - Level 3 - Projection.

2) Task and Goal Interdependence During Cooperation: In prior work, Gerkey and Matarić discuss constraints as they relate directly to interdependence: task type (singleversus multi-agent) and agent type (single versus multitask); these were further combined into a single schedulingbased interdependence definition in later work ([7]). The interdependence notion was further detailed by Phillips, Schaefer, Billings, et al., who identified the frequency of communication and task-flow interdependency during task execution as critical to characterizing the level of cooperation between them ([16]). The authors broke task-flow interdependence into three process structures: "parallel, "sequential, and "dialog-like. Their focus was on the process by which task execution unfolds, and the communication required between the working agents. Together, these dimensions more comprehensively categorize the overall interdependence during planning and execution stages, respectively.

Literature also elucidates a strong link between the projected criticality of a task (in terms of life-risk and importance to mission success) and the levels of robot autonomy within a mixed system ([6], [13]). Both works defined the level of robot autonomy as a spectrum of responsibility toward the mission. Beer, Fisk, and Rogers analyzed this via task breakdown and the allocation of stages to each or both agents, and outlined 10 specific configurations of these possible responsibility overlaps during task execution by deconstructing the task as a sense-plan-act cycle ([22]). Jiang and Arkin took a more goal-oriented view and used the overlap between the agents goals to assess those agents responsibilities. Our taxonomy includes task interdependence and its plan- and execution-time components in order to characterize the dynamics of an interaction. We label the level of robot autonomy as an effect of various contextual and local factors and discuss their interplay in Section $\mathrm{V}$

\section{Operational Contexts for Collaborative HRI WITH MOBILE ROBOTS}

Burke et al. ([1]) presented a list of applications and domains they deemed relevant to the HRI community; we

\footnotetext{
${ }^{1}$ In subsequent work, ([20]) comprehension was expanded to be of two kinds: identification of elements and interpretation of events within the current situation.
}

reviewed two of the listed operational contexts and observed three macro-themes that introduce complexity in these contexts: 1) environmental complexity, especially in terms of the risk involved; 2) knowledge of task goals and priorities or the task model; and 3) the differing nature of other agents or the team model. We chose the following scenarios in order to illustrate the variety of challenges that exist within the above dimensions. Though not exhaustive, they represent a range of challenges across the task, environment, and team dimensions.

\section{A. Urban Search and Rescue (USAR)}

The urban search and rescue (USAR) domain involves a search and rescue procedure performed within a potentially adversarial environment. (This domain is drawn partially from conversations with the Army Research Lab (ARL).)

In the USAR scenario, a heterogeneous team of human soldiers, unmanned aerial and ground vehicles, and autonomous aides work together to navigate an urban environment and perform a search-and-rescue operation within a building. This domain demonstrates complexity in terms of environment, task, and team. The environment is unknown a priori and is unstructured; it is also dynamic and adversarial in nature due to debris and adversarial agents operating within the area, posing a risk to both humans and autonomous agents. While these environmental factors place constraints on team operation (a concept discussed in work by Beer, Rieth, Tran, et al. [2]), explicit categorizations that adequately inform how these factors can impact team structure and interaction do not currently exist in the literature. Further, the USAR task is often complex and multistage, requiring planning, scouting, and execution ([7], [21]). Dynamic environmental factors can introduce constraints on possible approaches to the task online, and task and sub-task specifications may also require modification as additional information is acquired during execution. Also, team structures and hierarchies may be fluid and change during task execution, further increasing complexity. Finally, team members might not regularly work together, so gaps in mutual understanding may exist.

\section{B. Assembly Line}

In traditional manufacturing settings, robots operate behind fences, working apart from human beings on the manufacturing line and performing separate tasks. Now, manufacturing companies are interested in safe robots that are able to work in close proximity with human teammates; however, integrating robots in this manner introduces complexity in terms of both team and environmental factors. While tasks are well-defined, the environment and team are semistructured, requiring greater flexibility from robot teammates. The way in which a human-robot team approaches a given task will be more fluid, and robots will need to adapt to performing updated roles online if human teammates modify the way in which they approach their tasks. Additionally, an environment in which human workers are present is more dynamic and uncertain than the well-defined cage setting 
in which robots have previously operated. As in the USAR example, this scenario requires characterization of environmental factors, as well as a more thorough consideration of team roles and structure.

\section{GAP ANALYSIS}

In the previous section, we outlined the need for the following:

- A structure that can encompass both contextual factors and local dynamics while still preserving the differentiation between them.

- A combined synthesis of dimensions (task scheduling interdependence and task execution interdependence, for example) that incorporates differing perspectives of the surveyed taxonomies.

- Quantifiable role formulation grounded within a collective's shared knowledge structures, such that they can be used to assess and validate informational requirements at different levels of abstraction.

In the next section, we address the first two issues by proposing a reformulated upper ontology over the surveyed taxonomies. Specifically, we include the context-driven dimensions discussed earlier with the overarching context category further subdivided into three classes: task, team, and environment. These three classes are the result of our analysis of varying operational contexts (Section III) and identified dimensions from the review ([2]-[4], [8], [13]). The environment category is included as a major class despite not being explicitly characterized by existing taxonomies, as our analysis of operational contexts highlights environment as an important discriminator across civil, social, military, and commercial contexts. We also introduce an initial set of key factors for this class. The cooperation-driven taxonomic dimensions are included within the greater category of local dynamics, which is further broken down into task-work and team-work dynamics, inspired by the team- and task-work model used by behavioral scientists to study cooperative behaviors ([11]). We also address the second issue by placing the reviewed factors into this taxonomy, as well as synthesizing new categories relating the non-orthogonal factors from the surveyed papers.

\section{A ReFormed UPPER ONTOLOGY FOR ANALYZING HRI PROBLEMS}

Here, we propose our reformed upper ontology over the surveyed taxonomies. We situate these taxonomies within the proposed structure, which includes the following high-level categories: system context, local dynamics, and effects.

\section{A. SYSTEM CONTEXT}

Factors that fall into the system context category function as independent variables for a given situation and are taken as inputs to the HRI problem. Within this category, we identify contextual factors related to task, team, and environment and identify the additional subcategories of description and modeling within each group.

1) Task a) Description The following categorizations from the literature comprise the task description subcategory and define the task-related work that agents will engage in.

i) Task Nature ([16])- This category describes the type of requirement for a given task. A) Physical, B) Cognitive, and C) Social.

ii) Task Focus ([2])- For this category, we add a new entry $(*)$ to the list proposed by [2] in order to incorporate the training and learning phase of interactions as observed in domestic and other social domains. A) Transit, B) Area Coverage, C) Resource Management, D) Target Search, E) Construction, F) Assistance, and G) *Knowledge Acquisition 2

iii) Criticality $]^{3}([8],[13])$ - We divide this factor into two dimensions: criticality to mission success and criticality to human safety. Given the current state of robotic abilities and intelligence, the human safety axis should take precedence over the taskcritical axis when making decisions about tradeoffs between multiple action options. We suggest the following breakdown:
A) Low Criticality - The task is neither mission-critical nor affecting human safety.
B) Medium Criticality - The task is mission-critical, but does not affect human safety.
C) High Criticality - The task is critical to human safety.
D) Severe Criticality - The task is both mission- and safety-critical.

\section{2) Team}

a) Description Team description factors provide a way to define the diversity and capabilities of a mixed team in order to standardize the basic challenges and strengths of the collective.

i) Interaction Composition ([3], [4], [8]) This characterizes the diversity and numeric complexity of the team, aiding in the standardization of the communication mediums and protocols used by different compositions. It is a product of whether the interacting humans and robots are individuals (single) or acting as a group (multiple), and whether the interactants are homogeneous or heterogeneous in terms of goals, domain knowledge, decision functions, and possible actions ([17]). • Single Human to Single Robot • Single

${ }^{2}$ Examples of knowledge acquisition include learning the task model ([23], [24]) and learning the environment model ([25], [26]).

${ }^{3}$ Our ontology addresses this aspect at the macro level, but note that detailed frameworks exist ([27]) that provide a finer, more context-sensitive assessment. 
Human to Multiple-Homogeneous

Robots - Single Human to

Multi-Heterogeneous Robots

- Multi-Homogeneous Humans to

Single/Multi-Homo/Multi-Hetero

Robots - Multi-Heterogeneous Humans

to Single/Multi-Homo/Multi-Hetero Robots

ii) Capabilities This is a sample set of possible types of team member capabilities that can be used to standardize robot teammate functionalities. Note that specific categorizations for these types of capabilities will need to be identified in future work. ([3], [9], [17]): A) Motion B) Sensor C) Computation D) Communication

b) Modeling ([3], [9], [17]) - The following factors comprise the team modeling subcategory. They describe the models that team members have of their teammates' knowledge structures and decision functions. Specific categorizations will need to be identified in future work.

i) Shared-Knowledge Structures ([17])

ii) Decision Functions ([9], [17])

\section{3) Environment}

a) Description Environment description factors define the macro-aspects and byproducts of the deployment environment (such as physical space, bystander interactions, etc.).

i) Spatial Distribution ([5], [8], [12]) - Spatial relationships between agents have previously been modeled as a byproduct of the environment. For example, modern manufacturing entails a proximal configuration, while an office environment entails a hybrid configuration where some subtasks require physical proximity of teammates to a task and others can be executed remotely. The following are categorizations of spatial configurations: A) Proximal B) Hybrid C) Remote

b) Additional Taxonomy Suggestions. Here, we identify possible additional environment description categorizations beyond the ones identified in the literature:

i) Level of Cooperation - This category defines whether an environment is cooperative, neutral, or adversarial in terms of how environmental factors impact a team's progress towards its goals.

ii) Spatial and Temporal Complexity - This category defines whether an environment is static or dynamic in terms of physical features and whether the physical configuration is structured or unstructured.

iii) Mobility and Perception Constraints - In conjunction with an agent's motion and perception capabilities, this category should quantify the environmental constraints for each agent.

\section{B. LOCAL DYNAMICS}

Local dynamics variables are factors chosen to optimize team performance on a given task and can be broken down into two sub-categories: task and team ([11]). These factors impact how effectively a team can execute a task given the constraints imposed by contextual factors.

1) Task

a) Planning ([7], [21]) - We modify the "Instantaneous Assignment" and "Time-Extended" categorizations from Korsah, Stentz, and Dias [7] to three more general categories: online, hybrid and offline task planning.

b) Plan-Time Dependencies ([7], [21]) - We adopt the following categorizations for plan-time schedule dependencies from the iTax taxonomy in Korsah, Stentz, and Dias, Gerkey and Matarić [7], [21]: i) No Dependencies ii) In-Schedule Dependencies iii) Cross-Schedule Dependencies iv) Complex Dependencies

c) Run-Time Dependencies ([6], [13], [16]) - We define run-time dependencies as dependence between the "sense", "plan", and "act" stages of a task, as defined by Beer, Fisk, and Rogers [13]. Within each of these stages, task dependence can be described as joint or disjoint, and joint tasks can have parallel, sequential, or dialog-like process flows.

2) Team

a) Roles ([5], [15]) - We adopt the role categorizations outlined in Section II

b) Expertise Hierarchy ([3], [4], [8], [14]) - Roles are not always fixed in teams operating in complex environments. To capture the possibility of dynamic roles, we divide the expertise hierarchy category into two dimensions:

i) Reconfigurability - Team hierarchies can either remain fixed ([28], [29]) or be fluid ([30]).

ii) Decision-Making Protocol ([3], [4], [8]) - The decision-making structure within a team can be centralized with a single individual or agent, distributed to multiple teammates, or a hybrid of both.

c) Communication Structure ([3], [4], [17]) - Depending upon the perception constraint context, the nature of the task, and the communication capabilities of the team (as addressed in the System Context category), we adopt the following categories for communication structures:

i) None or Environment-Based ([3], [17]) In this case, no direct communication occurs between the interactants, and the agents are limited to observing changes in environment or a connecting physical medium in order to make inferences about other agents' actions and states.

ii) Sensing-Based ([3]) - Interactants can only communicate by directly sensing their teammates 
and observing their actions and states.

iii) Direct-Partial ([3], [4]) - Interactants have a communication channel, but are limited by a spatial or temporal range of communication.

iv) Direct-Full ([3], [4], [17]) - Interactants can communicate with any teammate located anywhere within the known environment.

\section{EFFECTS}

Effects are resultant factors driven by the operational context of a team, task, and environment, and the chosen dynamics for achieving a joint goal. Given the context and the task and team dynamics under our taxonomy, the level of autonomy of each teammate and the level of information abstraction for communicating with them should be set accordingly.

1) Level of Autonomy: We adopt the levels of automation defined by Beer, Fisk, and Rogers ([13]). In that work, automation levels are determined according to three modes of operation: "sense", "plan", and "act". Ten levels are delineated, assigning sense, plan, and act modes of operation to the human, the robot, or both. In our upper ontology, we expand the designation of human and robot roles in this classification to include any two teammates (human or autonomous) or subteams interacting within a larger team. Note that here, level of autonomy can be assigned to either a human or an autonomous agent.

Decisions about the level of autonomy are based upon which role a given teammate has assumed, and in what context. For example, an operator executing a task in a fully observable and fully modeled context might work at a higher level of autonomy than an operator executing the same task with a partial model or partial observability. In the latter case, the operator might rely more heavily upon teammates in order to gather and communicate necessary information and provide instruction.

2) Level of Information Abstraction. Grice's maxims [31] provide succinct guidelines for designing cooperative communication, and we wish to highlight two of those:

- "Maxim of Quantity - Be as informative as you can, and no more."

- "Maxim of Relation - Be relevant to the discussion."

The former maxim emphasises that during communication enough information should be provided such that the issue being discussed can be understood, however one should beware of information overload, i.e. communicating extra details which are irrelevant to the other person's mental model and can delay the uptake of information. The latter focuses on the relevance of the information being communicated to the issue being discussed. This indicates a strong inter-dependence between level of information abstraction, the issue being discussed, and the required situated awareness, for either agents, that support better decision-making or discussion. Assuming that agents interact rationally and discuss/act upon issues with the right experts, we can use team-roles under our taxonomy to provide us with the missing information about the mental models and their expertise. However, as has been hinted before in section IV an exact informational profile of a role would be needed to perform such assessments online, such that the information can be tailored to the nature of an interactant under the Maxim of Relation.

Following the thread of situational awareness ([10]), in order to make an informed decision about an issue it is important to know how much the interactants know about each other's current state and status. If the spatial relationship between agents is proximal, then interactants have direct access to physical situatedness; however, if their relationship is remote in nature, interactants are charged with exchanging information about both cognitive and physical situatedness. (By physical situatedness, we mean an understanding about each other's perception of the environment; by cognitive situatedness we mean understanding about each other's goals, plans and mission status.)

Finally, the level of information abstraction also depends upon the relevance of one interactants state to that of the other ([6], [13]). This relationship can be assessed along the dimensions of goal and task dependency, as outlined in our dynamics taxonomy. Our guidelines state that the greater the degree of interdependency, the finer the granularity of updates should be, conditioned upon the fact that the updates should contribute to dependency constraints in a positive or negative way. Similarly, depending upon whether the interactants have the same goal, the level of task abstraction should be adjusted such that the interactants share action or goallevel information, respectively.

\section{Discussion}

In this paper, we reviewed taxonomies and frameworks within the fields of human-robot interaction, algorithmic robotics systems, and behavioral sciences in order to better understand their influence on each other, to identify previously undocumented research gaps, and motivate future work. We observed not only that the factors identified by the surveyed taxonomies interact with each other, but that there also exists a top-down pattern in which higher-level factors affect the range of design choices over the factors below them. As a result of this analysis, we proposed an extensible taxonomy that breaks down an HRI system into three levels of depth: the system context-model, the local dynamics model of the interaction, and the resultant effects on leaf factors driving HRI. These levels are then used to characterize three major system components: task, environment and team. We arranged the reviewed taxonomies within this framework, using the insights gained by comparing across them to arrange similar factors across different dimensions (figure 1). 


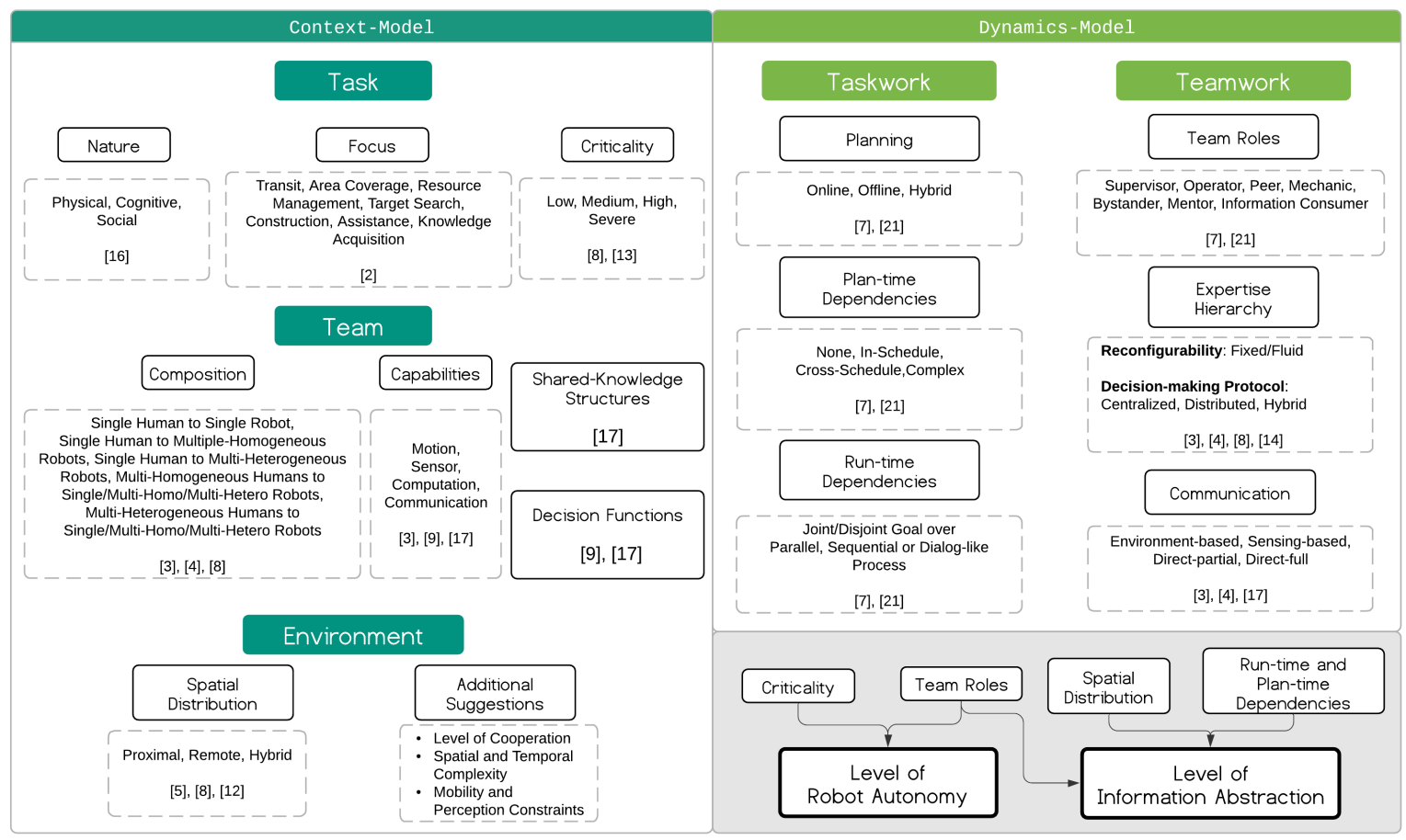

Fig. 1. Visualization for the Overarching Taxonomy. The columns on left and right provide an overview of the reviewed factors and how they are arranged in our taxonomy, both in terms of which component they belong to and their relative depth. The grey box in the right-bottom shows the effects and their interactions with the identified factors.

We observed two main areas of potential focus for future work. First, as we move toward "true" collaboration between humans and robots, there is a demonstrated need for enhanced communication protocols, flexible role changes, and adaptive communication for different kinds of human interactants (based on role, and situated context and awareness). This in turn underscores the need for a situational awareness assessment framework that is applicable to all three of the system components within our taxonomy. While the SAT model ([9]) grounded the principles of situational awareness as they apply to a systems mission specification, a space remains for the conceptualization of a framework that extends this idea to environment and team configurations as well. As per our survey, this would need to draw upon existing situational awareness principles ([10]) and a more structured understanding of potential human and robot roles ([5], [15]). Second, most of these taxonomies are formulated by making assumptions about the kinds of environments that they address. While this strengthens their characterizations for the specific interactions, this also means that:

A) These specific taxonomies do not include the environment as a major factor, and

B) There is a dearth of taxonomies, in general, that characterize the specific challenges of different operational environments.

This lack of explicit environment modeling results in an inability to capture environmental challenges that are important to design decisions. An example is obscuration of environment-based risk to the human operator in scenarios
III-A and III-B, which directly affects the recommended autonomy level or responsibility of the robot to perform the perception task. Additionally, lack of consideration about existent knowledge of semantic and physical mapping of the environment drastically affects the information exchange patterns in the USAR scenario in III-A This scenario requires both task and plan information to be exchanged for successful mission execution as well as information about the physical environment the team is operating in.

Existent literature additionally does not model the degree of observability of the environment or agent states, which makes it difficult to distinguish between information or context that is available and what additional situational data needs to be shared. For example, in the USAR context, teams are distributed over a large area and have limited observability of both the space and their teammates. Such a scenario demands that team members relate information about spaces, events, and their own state to others during task execution. The level of detail about the required information that should be shared between interactants is an important consideration according to the Maxim of Relation.

While our taxonomy serves as a first step toward defining challenges faces by HRI teams executing tasks in complex domains, more extensive characterization is still required. Such an investigation could facilitate the analysis of operational contexts for HRI in a standardized manner, allowing the insights from one taxonomy to be generalized to another. We note that this paper's length and scope restricted the detailed analysis of many aspects which affect interaction 
but have only been cursorily touched upon. The factors here mainly relate to structure of interaction between two agents or sub-teams, and do not focus on the pattern of that interaction. Additionally, some of the categories under the task, environment and team context do not have a complete enumeration of factors and quantified bins. In future work, it will be particularly important to expand upon factors related to environment description and modeling. Further, it will be important to expand upon the characterization of task modeling and team capabilities, including interdependence requirements. We hope that the insights from this survey and the taxonomic structure will facilitate the study of these issues in a systematic manner.

\section{REFERENCES}

[1] J. L. Burke, R. R. Murphy, E. Rogers, V. J. Lumelsky, and J. Scholtz, "Final report for the darpa/nsf interdisciplinary study on human-robot interaction," IEEE Transactions on Systems, Man, and Cybernetics, Part C (Applications and Reviews), vol. 34, no. 2, pp. 103-112, May 2004.

[2] R. Beer, C. A. Rieth, R. Tran, and M. B. Cook, "Framework for multi-human multi-robot interaction: Impact of operational context and team configuration on interaction task demands," in 2017 AAAI Spring Symposium Series, 2017.

[3] Y. U. Cao, A. S. Fukunaga, and A. Kahng, "Cooperative mobile robotics: Antecedents and directions," Autonomous Robots, vol. 4, no. 1, pp. 7-27, Mar. 1997.

[4] G. Dudek, M. R. M. Jenkin, E. Milios, and D. Wilkes, "A taxonomy for multi-agent robotics," Autonomous Robots, vol. 3, no. 4, pp. 375-397, Dec. 1996.

[5] M. A. Goodrich and A. C. Schultz, "Human-Robot Interaction: A Survey," Foundations and Trends ${ }^{\circledR}$ in HumanComputer Interaction, vol. 1, no. 3, pp. 203-275, 2007.

[6] S. Jiang and R. C. Arkin, "Mixed-initiative human-robot interaction: Definition, taxonomy, and survey," in 2015 IEEE International Conference on Systems, Man, and Cybernetics, Oct. 2015, pp. 954-961.

[7] G. A. Korsah, A. Stentz, and M. B. Dias, "A comprehensive taxonomy for multi-robot task allocation," Int. J. Rob. Res., vol. 32, no. 12, pp. 1495-1512, Oct. 2013.

[8] H. A. Yanco and J. Drury, "Classifying human-robot interaction: An updated taxonomy," in 2004 IEEE International Conference on Systems, Man and Cybernetics, vol. 3, Oct. 2004, 2841-2846 vol.3.

[9] J. Y. Chen, S. G. Lakhmani, K. Stowers, A. R. Selkowitz, J. L. Wright, and M. Barnes, "Situation awareness-based agent transparency and human-autonomy teaming effectiveness," Theoretical issues in ergonomics science, vol. 19, no. 3, pp. 259-282, 2018.

[10] M. R. Endsley, "Design and evaluation for situation awareness enhancement," in Proceedings of the Human Factors Society annual meeting, SAGE Publications Sage CA: Los Angeles, CA, vol. 32, 1988, pp. 97-101.

[11] J. Mathieu, T. S. Heffner, G. Goodwin, E. Salas, and J. Cannon-Bowers, "The Influence of Shared Mental Models on Team Process and Performance," Journal of Applied Psychology, vol. 85, pp. 273-283, 2000.

[12] T. Fong, I. R. Nourbakhsh, and K. Dautenhahn, "A survey of socially interactive robots.," Robotics and Autonomous Systems, vol. 42, no. 3-4, pp. 143-166, Mar. 1, 2006.

[13] J. M. Beer, A. D. Fisk, and W. A. Rogers, "Toward a framework for levels of robot autonomy in human-robot interaction.," Journal of human-robot interaction, vol. 32 , pp. 74-99, 2014.
[14] K. Dautenhahn, "Socially intelligent robots: Dimensions of human-robot interaction," Philosophical Transactions of the Royal Society of London B: Biological Sciences, vol. 362, no. 1480, pp. 679-704, 2007.

[15] J. Scholtz, "Theory and evaluation of human robot interactions," in 36th Annual Hawaii International Conference on System Sciences, 2003. Proceedings of the, vol. 24, IEEE, 2003, 10 pp. ISBN: 0-7695-1874-5.

[16] E. K. Phillips, K. Schaefer, D. R. Billings, F. Jentsch, and P. A. Hancock, "Human-Animal Teams as an Analog for Future Human-Robot Teams: Influencing Design and Fostering Trust," J. Human-Robot Interact., vol. 5, no. 1, p. 100 , Sep. 2015.

[17] P. Stone and M. Veloso, "Multiagent systems: A survey from a machine learning perspective," Autonomous Robots, vol. 8, no. 3, pp. 345-383, 2000.

[18] D. A. Norman and S. W. Draper, User Centered System Design; New Perspectives on Human-Computer Interaction. Hillsdale, NJ, USA: L. Erlbaum Associates Inc., 1986, ISBN: 0898597811.

[19] D. Feil-Seifer and M. Matarić, "Defining Socially Assistive Robotics," in 9th International Conference on Rehabilitation Robotics, 2005., IEEE, pp. 465-468, ISBN: 0-7803-9003-2.

[20] J. L. Burke, R. R. Murphy, M. D. Coovert, and D. Riddle, "Moonlight in miami: Field study of human-robot interaction in the context of an urban search and rescue disaster response training exercise," Human-Computer Interaction, vol. 19, pp. 85-116, 2004.

[21] B. P. Gerkey and M. J. Matarić, "A formal analysis and taxonomy of task allocation in multi-robot systems," The International Journal of Robotics Research, vol. 23, no. 9, pp. 939-954, 2004. eprint: https : / / doi . org / 10 . $1177 / 0278364904045564$.

[22] R. R. Murphy, Introduction to AI Robotics. MIT Press, 2000.

[23] T. Fitzgerald, A. Goel, and A. Thomaz, "Human-guided object mapping for task transfer," ACM Transactions on Human-Robot Interaction (THRI), vol. 7, no. 2, p. 17, 2018.

[24] M. Cakmak, N. DePalma, R. I. Arriaga, and A. L. Thomaz, "Exploiting social partners in robot learning," Autonomous Robots, vol. 29, no. 3, pp. 309-329, Nov. 2010.

[25] E. A. Topp and H. I. Christensen, "Topological modelling for human augmented mapping," in 2006 IEEE/RSJ International Conference on Intelligent Robots and Systems, IEEE, 2006, pp. 2257-2263.

[26] J. Peltason, F. H. Siepmann, T. P. Spexard, B. Wrede, M. Hanheide, and E. A. Topp, "Mixed-initiative in human augmented mapping," in 2009 IEEE International Conference on Robotics and Automation, IEEE, 2009, pp. 2146-2153.

[27] P. Lin, G. Bekey, and K. Abney, "Autonomous military robotics: Risk, ethics, and design," California Polytechnic State Univ San Luis Obispo, Tech. Rep., 2008.

[28] J. Casper and R. R. Murphy, "Human-robot interactions during the robot-assisted urban search and rescue response at the World Trade Center," IEEE Trans. Syst. Man, Cybern. Part B Cybern., vol. 33, no. 3, pp. 367-385, 2003.

[29] S. Thrun, M. Bennewitz, W. Burgard, A. B. Cremers, F. Dellaert, D. Fox, D. Hähnel, C. Rosenberg, N. Roy, J. Schulte, and D. Schulz, "MINERVA: a second-generation museum tour-guide robot," Proc. 1999 IEEE Int. Conf. Robot. Autom., no. May, pp. 1999-2005, 1999.

[30] A. Mörtl, M. Lawitzky, A. Kucukyilmaz, M. Sezgin, C. Basdogan, and S. Hirche, "The role of roles: Physical cooperation between humans and robots," The International Journal of Robotics Research, vol. 31, no. 13, pp. 1656-1674, 2012.

[31] H. P. Grice, "Logic and conversation," in Syntax and Semantics: Vol. 3: Speech Acts, P. Cole and J. L. Morgan, Eds., New York: Academic Press, 1975, pp. 41-58. 